\title{
A NEW DESCRIPTION AND ANALYSIS OF THE ARC SPECTRUM OF CHLORINE
}

\author{
By C. C. Kiess
}

\section{ABSTRACT}

New types of photographic plates have made it possible not only to extend our knowledge of the chlorine arc spectrum beyond the infrared limit hitherto reached, but also to record many new lines in the regions of shorter wave length. There are now presented wave lengths for nearly twice as many lines as have hitherto been known. The new data have made it possible to revise and improve the analysis of $\mathrm{Cl}$ I with the results: (1) Many new terms have been detected; (2) previous erroneous classifications have been corrected.

\section{CONTENTS}

I. Introduction ----

I. Introduction

III. Experimental procedure

IV. Acknowledgment.

\section{INTRODUCTION}

The description of the arc spectrum of chlorine presented in this paper constitutes an extension and revision of the work done four years ago at the Bureau of Standards by the writer in collaboration with T. L. deBruin. ${ }^{1}$ At that time, we were able to photograph the spectrum out to wave length 9,876 A with plates sensitized with the dyes then available. The wave-length data thus obtained made it possible to establish the main features of the term structure of $\mathrm{Cl}$ I and to determine the ionization potential of the neutral chlorine atom. Owing, however, to the limitations of the sensitized photographic plates employed in that work, not all the features of the spectrum predictable from atomic theory could be recorded. The discovery of new photo-sensitizing materials by the Research Laboratory of the Eastman Kodak Co. ${ }^{2}$ has made it desirable, therefore, to reinvestigate the arc spectrum of chlorine, both for the purpose of extending it into hitherto unexplored regions and to bring out with greater intensity those features which, in the previous work, had been recorded only fragmentarily. Since it will be necessary in this paper to refer frequently to the earlier paper, it will, hereafter, be briefly designated by RP73.

\section{EXPERIMENTAL PROCEDURE}

The light source used in the present work was a Geissler tube of the same type as that described in RP73. The chlorine generator of the new tube was charged, however, with palladium chloride, which is 
much less hygroscopic than the chloride of gold previously used. The chlorine vigorously attacked the hot platinum electrodes of the tube and had to be replenished from time to time until a state of equilibrium was attained, after which the tube was operated for over 200 hours without further admission of chlorine from the generator. The tube was excited by the uncondensed discharge from a 40,000-volt transformer.

The spectrograms were all obtained with the 21 -foot concave grating ruled with 7,500 lines per inch. The mounting of this instrument has been described elsewhere. ${ }^{3}$ Recently, however, the speed of this spectrograph has been considerably increased by substituting a collimating mirror of stainless steel for the tarnished speculum metal mirror. Nearly all the exposures to the first order infrared were made with only half the slit covered with a filter so that lines in the overlapping second order were also photographed. Both the first and second orders of the $\mathrm{Fe}$ arc spectrum were also photographed to supply the standards needed for the wave-length determinations. The exposures were of 24 hours duration for each setting of the spectrograph and covered the region from $3,000 \mathrm{~A}$ in the ultraviolet to $12,000 \mathrm{~A}$ in the infrared.

\section{RESULTS}

The new and revised wave lengths derived in this investigation are given in the first column of table 1. A total of 441 lines, believed to be characteristic of the arc spectrum of chlorine, is now listed as compared with the 225 lines reported in RP73. All the measurements made in the earlier and also the present series of observations have been combined into means which furnish a scale of wave lengths to which the new lines observed only on the recent spectrograms have been reduced. It is the results thus obtained that have been entered in the table. The intensities are estimates of the relative blackening produced by the lines, but owing to the variable spectral sensitivity of the plates, these estimates for groups of lines in different portions of the spectrum are not comparable with each other.

TABLE 1.-Wave lengths in the arc spectrum of chlorine

\begin{tabular}{|c|c|c|c|}
\hline$\lambda_{\text {air. I. A. }}$ & Intensity & $\nu_{\nabla \mathrm{aO}} \mathrm{cm}^{-1}$ & Term combination \\
\hline $10,427.52$ & 0 & $9,587.38$ & \\
\hline $10,420.19$ & 1 & $9,594.13$ & \\
\hline $10,392.45$ & 5 & $9,619.73$ & $4 s^{2} \mathrm{P}_{01 / 2}-4 p^{4} \mathrm{D}_{11 / 2}^{\circ}$ \\
\hline $10,320.00$ & 4 & $9,687.27$ & \\
\hline $10,315.28$ & & $9,691.70$ & \\
\hline $10,312.11$ & 1 & $9,694.69$ & \\
\hline $10,305.38$ & 0 & $9,701.01$ & \\
\hline $10,177.40$ & 1 & $9,823.00$ & $4 s^{2} \mathrm{P}_{01 / 2}-4 p^{4} \mathrm{D}_{01 / 2}$ \\
\hline $10,091.64$ & $\begin{array}{r}40 \\
1\end{array}$ & $9,906.48$ & $4 s^{2} \mathrm{P}_{11 / 2}-4 p^{4} \mathrm{D}_{21 / 2}^{2}$ \\
\hline & & & \\
\hline $\begin{array}{r}10,002.25 \\
9,897.79\end{array}$ & $\begin{array}{l}4 \\
3\end{array}$ & $\begin{array}{r}9,995.01 \\
10,100.50\end{array}$ & \\
\hline $9,875.95$ & 50 & $10,122.84$ & $4 s^{2} \mathrm{P}_{01 / 2}-4 p^{2} \mathrm{D}_{1 / 2}$ \\
\hline $\begin{array}{l}9,815.70 \\
9808,46\end{array}$ & $\frac{2}{5}$ & $10,184.97$ & \\
\hline $9,808.46$ & 5 & $10,192.49$ & $4 s^{2} D_{1 / 2}-4 p^{2} P_{1 / 2,2}^{1},\left(D^{1} D\right)$ \\
\hline
\end{tabular}

${ }^{3}$ B. S.Sci. Papers, vol. 18 (S441), p. 191, 1922. 
TABLE 1.-Wave lengths in the arc spectrum of chlorine-Continued

\begin{tabular}{|c|c|c|c|}
\hline$\lambda_{\mathrm{air}} . \mathrm{I} . \mathrm{A}$. & Intensity & $\nu_{\mathrm{VaO}} \mathrm{cm}^{-1}$ & Term combination \\
\hline $\begin{array}{l}9,806.90 \\
9,774.64 \\
9,744.33 \\
9,702.35 \\
9,669.48\end{array}$ & $\begin{array}{r}25 \\
1 \\
30 \\
40 \\
1\end{array}$ & $\begin{array}{l}10,194.11 \\
10,227.75 \\
10,259.57 \\
10,303.96 \\
10,338.98\end{array}$ & $\begin{array}{l}\left.4 s^{2} \mathrm{D}_{2 / / 2}-4 p^{2} \mathrm{P}_{1 / 2,}^{\circ}\left({ }^{1} \mathrm{I}^{1} \mathrm{I}\right)\right) \\
4 s^{2} \mathrm{P}_{1 / 2}-4 p^{4} \mathrm{D}_{1 / 5}^{\circ} \\
4 s^{4} \mathrm{P}_{01 / 2}-4 p^{4} \mathrm{P}_{1 / 5}^{\circ}\end{array}$ \\
\hline $\begin{array}{l}9,661.90 \\
9,632.37 \\
9,608.98 \\
9,592.20 \\
9,584.77\end{array}$ & $\begin{array}{r}20 \\
20 \\
4 \\
75 \\
50\end{array}$ & $\begin{array}{l}10,347.20 \\
10,378.81 \\
10,404.08 \\
10,422.28 \\
10,430.36\end{array}$ & $\begin{array}{l}\left.4 s^{2} \mathrm{D}_{11 / 2}-4 p^{2} \mathrm{P}_{01 / 2,}^{\circ},\left({ }^{1} \mathrm{I}\right)^{1} \mathrm{D}\right) \\
4 s^{2} \mathrm{P}_{01 / 2}-4 p^{2} \mathrm{~S}_{01 / 5}^{0} \\
4 s^{2} \mathrm{P}_{11 / 2}-4 p^{2} \mathrm{D}_{21 / 5}^{\circ} \\
4 s^{4} \mathrm{P}_{11 / 2}-4 p^{4} \mathrm{P}_{21 / 2}^{2}\end{array}$ \\
\hline $\begin{array}{l}9,554.96 \\
9,486.89 \\
9,481.93 \\
9,452.06 \\
9,419.74\end{array}$ & $\begin{array}{r}4 \\
25 \\
3 \\
75 \\
2\end{array}$ & $\begin{array}{l}10,462.90 \\
10,537.97 \\
10,543.49 \\
10,576.80 \\
10,613.10\end{array}$ & $\begin{array}{l}4 s^{2} \mathrm{P}_{11 / 2}-4 p^{4} \mathrm{D}_{01 / 2}^{\circ} \\
4 s^{4} \mathrm{P}_{01 / 2}-4 p^{4} \mathrm{P}_{01 / 2}^{0} \\
4 s^{2} \mathrm{D}_{21 / 2}-5 p^{4} \mathrm{P}_{1 / 2,2}^{\circ}\left({ }^{1} \mathrm{D}^{3} I^{\prime}\right) \\
4 s^{2} \mathrm{P}_{01 / 2}-4 p^{2} \mathrm{P}_{11 / 2}^{\circ} \\
4 s^{2} \mathrm{D}_{21 / 2}-5 p^{4} \mathrm{D}_{31 / 2}^{\circ},\left({ }^{1} \mathrm{D}^{3} \mathrm{P}\right)\end{array}$ \\
\hline $\begin{array}{l}9,400.76 \\
9,393.81 \\
9,371 \cdot 92 \\
9,288.82 \\
9,212.39\end{array}$ & $\begin{array}{r}1 \\
50 \\
0 \\
60 \\
3\end{array}$ & $\begin{array}{l}10,634.65 \\
10,642.39 \\
10,667.36 \\
10,762.68 \\
10,851.98\end{array}$ & $\begin{array}{l}4 s^{4} \mathrm{P}_{11 / 2}-4 p^{4} \mathrm{P}_{1 / 2 / 2}^{\circ} \\
4 s^{2} \mathrm{P}_{1 / 2}-4 p^{2} \mathrm{D}_{1 / 2}^{\circ} \\
4 s^{2} \mathrm{D}_{1 / 2}-5 p^{4} \mathrm{P}_{01 / 21}^{\circ},\left({ }^{1} \mathrm{D}^{3} \mathrm{P}\right)\end{array}$ \\
\hline $\begin{array}{l}9,197.49 \\
9,191.67 \\
9,121.10 \\
9,073.15 \\
9,069.66\end{array}$ & $\begin{array}{l}25 \\
60 \\
75 \\
50 \\
25\end{array}$ & $\begin{array}{l}10,869.56 \\
10,876.44 \\
10,960.58 \\
11,018.50 \\
11,022.74\end{array}$ & $\begin{array}{l}4 s^{2} \mathrm{P}_{01 / 2}-4 p^{4} \mathrm{~S}_{11 / 2}^{\circ} \\
4 s^{4} \mathrm{P}_{11 / 2}-4 p^{4} \mathrm{P}_{01 / 2}^{\circ} \\
4 s^{4} \mathrm{P}_{21 / 2}-4 p^{4} \mathrm{P}_{21 / 2}^{\circ} \\
4 s^{2} \mathrm{P}_{11 / 2}-4 p^{2} \mathrm{~S}_{01 / 2}^{\circ} \\
4 s^{2} \mathrm{D}_{11 / 2}-4 p^{2} \mathrm{~F}_{21 / 2}^{\circ},\left({ }^{1} \mathrm{D}^{1} \mathrm{D}\right)\end{array}$ \\
\hline $\begin{array}{l}9,068.39 \\
9,045.40 \\
9,038.96 \\
8,948.01 \\
8,931.20\end{array}$ & $\begin{array}{r}3 \\
40 \\
30 \\
50 \\
2\end{array}$ & $\begin{array}{l}11,024.30 \\
11,052.31 \\
11,060.31 \\
11,172.60 \\
11,193.63\end{array}$ & $\begin{array}{l}4 s^{2} \mathrm{D}_{21 / 2}-4 p^{2} \mathrm{~F}_{21 / 2,}^{0},\left({ }^{1} \mathrm{D}^{1} \mathrm{D}\right) \\
4 s^{2} \mathrm{P}_{01 / 2}-4 p^{2} \mathrm{P}_{01 / 2} \\
4 s^{2} \mathrm{D}_{21 / 2}-4 p^{2} \mathrm{~F}_{31 / 2}^{3},\left({ }^{1} \mathrm{D}^{1} \mathrm{D}\right) \\
4 s^{4} \mathrm{P}_{21 / 2}-4 p^{4} \mathrm{P}_{11 / 2} \\
4 s^{2} \mathrm{D}_{21 / 2}-5 p^{4} \mathrm{D}_{1 / 23}^{1},\left({ }^{1} \mathrm{D}^{3} \mathrm{P}\right)\end{array}$ \\
\hline $\begin{array}{l}8,918.78 \\
8,912.88 \\
8,846.00 \\
8,815.26 \\
8,759.93\end{array}$ & $\begin{array}{r}0 \\
40 \\
1 \\
0 \\
0\end{array}$ & $\begin{array}{l}11,209.36 \\
11,216.79 \\
11,301.44 \\
11,340.85 \\
11,412.49\end{array}$ & $\begin{array}{l}4 p^{4} \mathrm{D}_{01 / 2}^{\circ}-4 d^{4} \mathrm{D}_{11 / 2} \\
4 s^{2} \mathrm{P}_{11 / 2}-4 p^{2} \mathrm{P}_{11 / 2}^{\circ} \\
4 p^{4} \mathrm{D}_{1 / 2}^{\circ}-4 d^{4} \mathrm{D}_{21 / 2} \\
4 p^{4} \mathrm{D}_{11 / 2}^{\circ}-4 d^{4} \mathrm{D}_{11 / 2}\end{array}$ \\
\hline $\begin{array}{l}8,711.58 \\
8,686.28 \\
8,641.75 \\
8,628.61 \\
8,585.96\end{array}$ & $\begin{array}{r}1 \\
30 \\
3 \\
4 \\
100\end{array}$ & $\begin{array}{l}11,475.83 \\
11,509.25 \\
11,568.56 \\
11,586.17 \\
11,643.72\end{array}$ & $\begin{array}{l}4 s^{2} \mathrm{D}_{11 / 2}-5 p^{2} \mathrm{~S}_{01 / 2}^{\circ},\left({ }^{1} \mathrm{I}^{3} \mathrm{P}\right) \\
4 s^{2} \mathrm{P}_{11 / 2}-4 p^{4} \mathrm{~S}_{11 / 2}^{\circ} \\
4 p^{4} \mathrm{D}_{21 / 2}^{\circ}-4 d^{4} \mathrm{D}_{31 / 2}^{\circ} \\
4 s^{2} \mathrm{D}_{21 / 2}-5 p^{2} \mathrm{D}_{11 / 2}^{\circ}\left({ }^{1} \mathrm{I}^{3} \mathrm{I}^{\prime}\right) \\
4 s^{4} \mathrm{P}_{11 / 2}-4 p^{4} \mathrm{D}_{21 / 2}^{\circ}\end{array}$ \\
\hline $\begin{array}{l}8,577.98 \\
8,575.25 \\
8,550.46 \\
8,497.32 \\
8,494.52\end{array}$ & $\begin{array}{r}7 \\
75 \\
20 \\
5 \\
0\end{array}$ & $\begin{array}{l}11,654.56 \\
11,658.27 \\
11,692.07 \\
11,765.19 \\
11,769.07\end{array}$ & $\begin{array}{l}4 p^{4} \mathrm{D}_{21 / 2}^{\circ}-4 d^{4} \mathrm{D}_{23 / 2} \\
4 s^{4} \mathrm{P}_{03 / 2}-4 p^{4} \mathrm{D}_{11 / 2}^{\circ} \\
4 s^{2} \mathrm{P}_{13 / 2}-4 p^{2} \mathrm{P}_{01 / 2}^{\circ} \\
4 p^{4} \mathrm{D}_{21 / 2}^{\circ}-4 d^{4} \mathrm{D}_{11 / 2}\end{array}$ \\
\hline $\begin{array}{l}8,472.96 \\
8,467.32 \\
8,428.25 \\
8,422.20 \\
8,406.20\end{array}$ & $\begin{array}{r}3 \\
25 \\
100 \\
2 \\
10\end{array}$ & $\begin{array}{l}11,799.01 \\
11,806.87 \\
11,861.60 \\
11,870.12 \\
11,892.72\end{array}$ & $\begin{array}{l}4 p^{4} \mathrm{~S}_{11 / 2}-4 d^{2} \mathrm{D}_{21 / 2} \\
4 p^{4} \mathrm{D}_{31 / 2}^{1}-4 d^{4} \mathrm{D}_{31 / 2} \\
4 s^{4} \mathrm{P}_{01 / 2}-4 p^{4} \mathrm{D}_{01 / 2}^{0} \\
4 p^{4} \mathrm{D}_{31 / 2}^{\circ}-4 d^{4} \mathrm{D}_{21 / 2}\end{array}$ \\
\hline
\end{tabular}


TABLE 1.-Wave lengths in the arc spectrum of chlorine-Continued

\begin{tabular}{|c|c|c|c|}
\hline$\lambda_{\mathrm{Bir}}$. I. A. & Intensity & $\nu_{\mathrm{VaO}} \mathrm{cm}^{-1}$ & Term combination \\
\hline $\begin{array}{l}8,403.70 \\
8,397.61 \\
8,392.48 \\
8,383.58 \\
8,380.54\end{array}$ & $\begin{array}{l}1 \\
3 \\
2 \\
4 \\
2\end{array}$ & $\begin{array}{l}11,896.25 \\
11,904.88 \\
11,912.16 \\
11,924.81 \\
11,929.13\end{array}$ & $4 p^{2} \mathrm{P}_{11 / 2}^{\circ}-4 d^{4} \mathrm{P}_{21 / 2}$ \\
\hline $\begin{array}{l}8,375.95 \\
8,355.38 \\
8,343.97 \\
8,333.29 \\
8,330.57\end{array}$ & $\begin{array}{r}150 \\
1 \\
4 \\
100 \\
0\end{array}$ & $\begin{array}{l}11,935.66 \\
11,965.05 \\
11,981.41 \\
11,996.77 \\
12,000.69\end{array}$ & $\begin{array}{l}4 s^{4} \mathrm{P}_{21 / 2}-4 p^{4} \mathrm{D}_{31 / 2}^{\circ} \\
4 s^{4} \mathrm{P}_{11 / 2}-4 p^{4} \mathrm{D}_{11 / 2}^{\circ}\end{array}$ \\
\hline $\begin{array}{l}8,321.43 \\
8,311.15 \\
8,304.69 \\
8,286.67 \\
8,280.95\end{array}$ & $\begin{array}{l}1 \\
1 \\
5 \\
5 \\
7\end{array}$ & $\begin{array}{l}12,013.87 \\
12,028.73 \\
12,038.08 \\
12,064.26 \\
12,072.59\end{array}$ & $\begin{array}{l}4 p^{2} \mathrm{P}_{11 / 2}^{\circ}-6 s^{4} \mathrm{P}_{11 / 2} \\
4 p^{4} \mathrm{~S}_{11 / 2}^{\circ}-4 d^{2} \mathrm{D}_{11 / 2}\end{array}$ \\
\hline $\begin{array}{l}8,273.79 \\
8,271.70 \\
8,269.15 \\
8,267.97 \\
8,258.45\end{array}$ & $\begin{array}{r}7 \\
7 \\
10 \\
3 \\
5\end{array}$ & $\begin{array}{l}12,083.04 \\
12,086.10 \\
12,089.82 \\
12,091.55 \\
12,105.48\end{array}$ & $\begin{array}{l}4 p^{2} \mathrm{D}_{21 / 2}^{\circ}-4 d^{4} \mathrm{~F}_{31 / 2} \\
4 p^{2} \mathrm{P}_{11 / 2}^{\circ}-4 d^{2} \mathrm{D}_{21 / 2}\end{array}$ \\
\hline $\begin{array}{l}8,255.70 \\
8,244.50 \\
8,226.60 \\
8,221.73 \\
8,220.40\end{array}$ & $\begin{array}{r}1 \\
1 \\
2 \\
75 \\
60\end{array}$ & $\begin{array}{l}12,109.52 \\
12,125.97 \\
12,152.35 \\
12,159.55 \\
12,161.52\end{array}$ & $\begin{array}{l}4 s^{4} \mathrm{P}_{11 / 2}-4 p^{2} \mathrm{D}_{21 / 2}^{0} \\
4 s^{4} \mathrm{P}_{01 / 2}-4 p^{2} \mathrm{D}_{11 / 2}^{0}\end{array}$ \\
\hline $\begin{array}{l}8,212.00 \\
8,206.40 \\
8,203.76 \\
8,200.20 \\
8,199.02\end{array}$ & $\begin{array}{r}100 \\
2 \\
12 \\
35 \\
35\end{array}$ & $\begin{array}{l}12,173.96 \\
12,182.27 \\
12,186.19 \\
12,191.48 \\
12,193.23\end{array}$ & $\begin{array}{l}4 s^{4} \mathrm{P}_{21 / 2}-4 p^{4} \mathrm{D}_{21 / 2}^{\circ} \\
4 p^{2} \mathrm{P}_{01 / 2}^{\circ}-6 s^{4} \mathrm{P}_{01 / 2} \\
4 p^{2} \mathrm{D}_{21 / 2}^{\circ}-4 d^{2} \mathrm{~F}_{31 / 2}^{\circ} \\
4 s^{2} \mathrm{D}_{11 / 2}-5 p^{2} \mathrm{P}_{11 / 2}^{\circ},\left({ }^{1} \mathrm{D}^{3} \mathrm{P}\right) \\
4 s^{2} \mathrm{D}_{21 / 2}-5 p^{2} \mathrm{P}_{11 / 2}^{\circ},\left({ }^{1} \mathrm{D}^{3} \mathrm{P}\right)\end{array}$ \\
\hline $\begin{array}{l}8,194.35 \\
8,175.24 \\
8,170.09 \\
8,164.76 \\
8,161.52\end{array}$ & $\begin{array}{r}50 \\
0 \\
10 \\
1 \\
4\end{array}$ & $\begin{array}{l}12,200.18 \\
12,228.70 \\
12,236.40 \\
12,244.40 \\
12,249.26\end{array}$ & $\begin{array}{l}4 s^{4} \mathrm{P}_{11 / 2}-4 p^{4} \mathrm{D}_{01 / 2}^{\circ} \\
4 p^{2} \mathrm{~S}_{01 / 2}^{\circ}-6 s^{4} \mathrm{P}_{11 / 2} \\
4 p^{2} \mathrm{D}_{11 / 2}^{\circ}-6 s^{4} \mathrm{P}_{21 / 2}\end{array}$ \\
\hline $\begin{array}{l}8,129.55 \\
8,121.40 \\
8,117.75 \\
8,115.40 \\
8,094.76\end{array}$ & $\begin{array}{r}2 \\
5 \\
4 \\
2 \\
12\end{array}$ & $\begin{array}{l}12,297.43 \\
12,309.76 \\
12,315.30 \\
12,318.87 \\
12,350.28\end{array}$ & $\begin{array}{l}4 p^{2} \mathrm{D}_{21 / 2}^{\circ}-4 d^{4} \mathrm{~F}_{21 / 2} \\
4 p^{4} \mathrm{~S}_{11 / 2}-4 d^{4} \mathrm{P}_{11 / 2} \\
4 p^{2} \mathrm{D}_{11 / 2}^{\circ}-4 d^{4} \mathrm{P}_{21 / 2}\end{array}$ \\
\hline $\begin{array}{l}8,087.69 \\
8,086.67 \\
8,085.54 \\
8,084.48 \\
8,051.08\end{array}$ & $\begin{array}{l}20 \\
75 \\
60 \\
35 \\
20\end{array}$ & $\begin{array}{l}12,361.08 \\
12,362.63 \\
12,364.36 \\
12,365.98 \\
12,417.28\end{array}$ & $\begin{array}{l}4 s^{2} \mathrm{D}_{11 / 2}-4 p^{2} \mathrm{D}_{21 / 2}^{\circ},\left({ }^{1} \mathrm{D}^{1} \mathrm{D}\right) \\
4 s^{2} \mathrm{D}_{21 / 2}-4 p^{2} \mathrm{D}_{21 / 2}^{\circ},\left({ }^{1} \mathrm{D}^{1} \mathrm{D}\right) \\
4 s^{2} \mathrm{D}_{11 / 2}-4 p^{2} \mathrm{D}_{11 / 2}^{11},\left({ }^{1} \mathrm{D}^{1} \mathrm{D}\right) \\
4 s^{2} \mathrm{D}_{21 / 2}-4 p^{2} \mathrm{D}_{11 / 2}^{\circ},\left({ }^{1} \mathrm{D}^{1} \mathrm{D}\right) \\
4 s^{4} \mathrm{P}_{01 / 2}-4 p^{2} \mathrm{~S}_{01 / 2}^{\circ}\end{array}$ \\
\hline $\begin{array}{l}8,044.65 \\
8,023.30 \\
8,015.57 \\
7,997.80 \\
7,989.36\end{array}$ & $\begin{array}{r}0 \\
18 \\
45 \\
50 \\
0\end{array}$ & $\begin{array}{l}12,427.20 \\
12,460.28 \\
12,472.31 \\
12,500.01 \\
12,513.21\end{array}$ & $\begin{array}{l}4 p^{4} \mathrm{D}_{11 / 2}-4 d^{4} \mathrm{~F}_{21 / 2} \\
4 s^{2} \mathrm{D}_{11 / 2}-5 p^{2} \mathrm{P}_{01 / 2}^{\circ},\left({ }^{1} \mathrm{D}^{3} \mathrm{P}\right) \\
4 s^{4} \mathrm{P}_{13 / 2}-4 p^{2} \mathrm{D}_{11 / 2}^{\circ}\end{array}$ \\
\hline
\end{tabular}


TABLE 1.-Wave lengths in the arc spectrum of chlorine-Continued

\begin{tabular}{|c|c|c|c|}
\hline$\lambda_{\text {sir. I. A. }}$ & Intensity & $\nu_{\mathrm{V} \mathbf{~} \mathrm{O}} \mathrm{cm}^{-1}$ & Term combination \\
\hline $\begin{array}{l}7,985.80 \\
7,985.10 \\
7,980.58 \\
7,976.95 \\
7,974.72\end{array}$ & $\begin{array}{r}4 \\
1 \\
15 \\
25 \\
20\end{array}$ & $\begin{array}{l}12,518.79 \\
12,519.88 \\
12,526.97 \\
12,532.58 \\
12,536.18\end{array}$ & 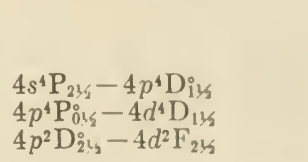 \\
\hline $\begin{array}{l}7,968.66 \\
7,962.12 \\
7,952.49 \\
7,940.65 \\
7,938.90\end{array}$ & $\begin{array}{r}3 \\
0 \\
15 \\
2 \\
8\end{array}$ & $\begin{array}{l}12,545.71 \\
12,556.02 \\
12,571.22 \\
12,589.97 \\
12,592.74\end{array}$ & $\begin{array}{l}4 p^{2} \mathrm{D}_{1, \zeta}-4 d^{2} \mathrm{D}_{21 / \zeta} \\
4 p^{4} \mathrm{D}_{01 / 5}^{2}-4 d^{4} \mathrm{~F}_{1 / \zeta} \\
4 p^{2} \mathrm{D}_{21 / 2}-6 s^{4} \mathrm{P}_{2 \zeta / 5}\end{array}$ \\
\hline $\begin{array}{l}7,935.00 \\
7,933.85 \\
7,924.62 \\
7,915.09 \\
7,899.28\end{array}$ & $\begin{array}{r}40 \\
50 \\
100 \\
25 \\
45\end{array}$ & $\begin{array}{l}12,598.93 \\
12,600.76 \\
12,615.44 \\
12,630.62 \\
12,655.90\end{array}$ & 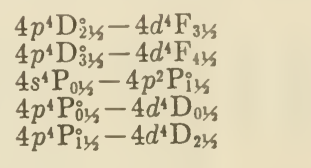 \\
\hline $\begin{array}{l}7,898.10 \\
7,893.33 \\
7,886.00 \\
7,878.22 \\
7,872.50\end{array}$ & $\begin{array}{r}5 \\
10 \\
6 \\
75 \\
1\end{array}$ & $\begin{array}{l}12,657.80 \\
12,665.45 \\
12,677.22 \\
12,689.74 \\
12,698.96\end{array}$ & $\begin{array}{l}4 p^{2} \mathrm{P}_{1 \zeta \zeta}^{\circ}-6 s^{4} \mathrm{P}_{01 / 5} \\
4 s^{4} \mathrm{P}_{21 / 5}-4 p^{2} \mathrm{D}_{21 / 5} \\
4 p^{4} \mathrm{D}_{1 \zeta / 5}^{\circ}-4 d^{2} \mathrm{~F}_{21 / 5}^{\circ}\end{array}$ \\
\hline $\begin{array}{l}7,870.68 \\
7,839.42 \\
7,837.40 \\
7,830.76 \\
7,825.80\end{array}$ & $\begin{array}{r}1 \\
8 \\
6 \\
30 \\
3\end{array}$ & $\begin{array}{l}12,701.90 \\
12,752.54 \\
12,755.83 \\
12,766.64 \\
12,774.74\end{array}$ & 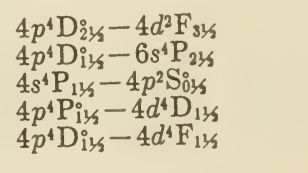 \\
\hline $\begin{array}{l}7,821.35 \\
7,815.34 \\
7,810.06 \\
7,805.82 \\
7,804.25\end{array}$ & $\begin{array}{r}45 \\
1 \\
3 \\
1 \\
2\end{array}$ & $\begin{array}{l}12,782.01 \\
12,791.83 \\
12,799.60 \\
12,807.44 \\
12,810.01\end{array}$ & $\begin{array}{l}4 p^{4} \mathrm{P}_{21 / 5}^{\circ}-4 d^{4} \mathrm{D}_{31 / 5} \\
4 p^{4} \mathrm{D}_{015,}^{\circ}-6 s^{4} \mathrm{P}_{1 \zeta / 5} \\
4 p^{2} \mathrm{~S}_{015}^{\circ}-4 d^{4} \mathrm{P}_{13 / 5}^{\circ}\end{array}$ \\
\hline $\begin{array}{l}7,802.27 \\
7,798.59 \\
7,790.56 \\
7,787.75 \\
7,777.82\end{array}$ & $\begin{array}{r}6 \\
5 \\
5 \\
4 \\
10\end{array}$ & $\begin{array}{l}12,813.26 \\
12,819.31 \\
12,832.52 \\
12,837.15 \\
12,853.54\end{array}$ & $\begin{array}{l}4 p^{4} \mathrm{D}_{213 /}-4 d^{4} \mathrm{~F}_{21 / 5} \\
4 p^{2} \mathrm{D}_{1 / 5}^{\circ}-4 d^{2} \mathrm{D}_{13 / 5} \\
4 p^{2} \mathrm{D}_{21 / 5}^{\circ}-6 s^{4} \mathrm{P}_{11 / 5} \\
4 p^{4} \mathrm{D}_{31 / 5}^{\circ}-4 d^{4} \mathrm{~F}_{31 / 5}^{\circ} \\
4 p^{4} \mathrm{D}_{135}^{\circ}-4 d^{4} \mathrm{P}_{21 / 5}^{\circ}\end{array}$ \\
\hline $\begin{array}{l}7,771.10 \\
7,769.18 \\
7,758.14 \\
7,754.78 \\
7,744.94\end{array}$ & $\begin{array}{r}12 \\
30 \\
3 \\
6 \\
125\end{array}$ & $\begin{array}{l}12,864.65 \\
12,867.83 \\
12,886.15 \\
12,891.73 \\
12,908.11\end{array}$ & 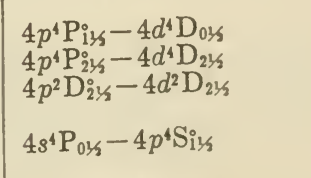 \\
\hline $\begin{array}{l}7,717.57 \\
7,714.56 \\
7,702.89 \\
7,697.40 \\
7,692.97\end{array}$ & $\begin{array}{r}100 \\
0 \\
10 \\
2 \\
7\end{array}$ & $\begin{array}{l}12,953.89 \\
12,958.94 \\
12,978.57 \\
12,987.83 \\
12,995.31\end{array}$ & $\begin{array}{l}4 s^{4} \mathrm{P}_{11 / 3}-4 p^{2} \mathrm{P}_{11 / 2} \\
4 p^{4} \mathrm{P}_{21 / 5}^{\circ}-4 d^{4} \mathrm{D}_{11 / 2} \\
4 p^{4} \mathrm{D}_{11 / 2}^{\circ}-6 s^{4} \mathrm{P}_{11 / 2}\end{array}$ \\
\hline $\begin{array}{l}7,672.44 \\
7,664.35\end{array}$ & $\begin{array}{r}25 \\
1\end{array}$ & $\begin{array}{l}13,030.08 \\
13,043.83\end{array}$ & $4 s^{4} \mathrm{P}_{21 / 5}-4 p^{2} \mathrm{D}_{1 / 3}$ \\
\hline $7,659.50$ & 5 & $13,052.10$ & $\left\{\begin{array}{l}4 p^{4} \mathrm{D}_{21 / 5}^{\circ}-4 d^{2} \mathrm{~F}_{21 / 3} \\
4 p^{4} \mathrm{D}_{335}^{2}-4 d^{4} \mathrm{~F}_{21 / 2}\end{array}\right.$ \\
\hline $7,656.86$ & 6 & $13,056.59$ & $4 p^{2} \mathrm{D}_{1 / 5 / 2}-4 d^{4} \mathrm{P}_{1 / 3 /}$ \\
\hline
\end{tabular}


TABLE 1.-Wave lengths in the arc spectrum of chlorine-Continued

\begin{tabular}{|c|c|c|c|}
\hline$\lambda_{\mathrm{air}}$. I. A. & Intensity & $\nu_{\mathrm{raO}} \mathrm{cm}^{-1}$ & Term combination \\
\hline $\begin{array}{l}7,635.08 \\
7,633.83 \\
7,628.35 \\
7,600.05 \\
7,561.19\end{array}$ & $\begin{array}{l}1 \\
3 \\
1 \\
0 \\
4\end{array}$ & $\begin{array}{l}13,092.30 \\
13,096.00 \\
13,105.29 \\
13,154.19 \\
13,221.80\end{array}$ & $4 p^{4} \mathrm{D}_{21 / 2}^{\circ}-6 s^{4} \mathrm{P}_{21 / 2}$ \\
\hline $\begin{array}{l}7,547.06 \\
7,503.80 \\
7,496.48 \\
7,492.12 \\
7,489.46\end{array}$ & $\begin{array}{r}100 \\
0 \\
1 \\
10 \\
8\end{array}$ & $\begin{array}{l}13,246.55 \\
13,322.92 \\
13,335.93 \\
13,343.69 \\
13,348.43\end{array}$ & $\begin{array}{l}4 s^{4} \mathrm{P}_{11 / 2}-4 p^{4} \mathrm{~S}_{11 / 5}^{\circ} \\
4 p^{4} \mathrm{D}_{11 / 2}^{\circ}-4 d^{2} \mathrm{D}_{11 / 2} \\
4 p^{4} \mathrm{D}_{31 / 2}^{\circ}-6 s^{4} \mathrm{P}_{21 / 2} \\
4 p^{4} \mathrm{D}_{21 / 2}^{\circ}-6 s^{4} \mathrm{P}_{11 / 2}^{\circ}\end{array}$ \\
\hline $\begin{array}{l}7,462.40 \\
7,459.42 \\
7,454.08 \\
7,444.32 \\
7,437.37\end{array}$ & $\begin{array}{l}8 \\
3 \\
2 \\
3 \\
1\end{array}$ & $\begin{array}{l}13,396.83 \\
13,402.19 \\
13,410.49 \\
13,429.37 \\
13,441.92\end{array}$ & $\begin{array}{l}4 p^{2} \mathrm{D}_{21 / 2}^{\circ}-4 d^{4} \mathrm{P}_{11 / 2} \\
4 p^{4} \mathrm{D}_{21 / 2}^{2}-4 d^{2} \mathrm{D}_{21 / 2} \\
4 p^{4} \mathrm{D}_{01 / 2}^{\circ}-6 s^{4} \mathrm{P}_{01 / 2} \\
4 s^{4} \mathrm{P}_{11 / 2}-4 p^{2} \mathrm{P}_{01 / 2}^{\circ}\end{array}$ \\
\hline $\begin{array}{l}7,435.71 \\
7,431.33 \\
7,414.10 \\
7,382.47 \\
7,372.65\end{array}$ & $\begin{array}{r}7 \\
0 \\
90 \\
3 \\
1\end{array}$ & $\begin{array}{l}13,444.91 \\
13,452.84 \\
13,484.10 \\
13,541.88 \\
13,559.91\end{array}$ & $\begin{array}{l}4 p^{4} \mathrm{D}_{31 / 2}^{\circ}-4 d^{4} \mathrm{P}_{21 / 2} \\
4 s^{4} \mathrm{P}_{21 / 2}-4 p^{2} \mathrm{P}_{11 / 2}^{\circ} \\
4 p^{4} \mathrm{D}_{11 / 2}^{\circ}-4 d^{4} \mathrm{P}_{11 / 2}\end{array}$ \\
\hline $\begin{array}{l}7,342.74 \\
7,342.00 \\
7,329.33 \\
7,296.38 \\
7,256.63\end{array}$ & $\begin{array}{r}3 \\
1 \\
3 \\
1 \\
125\end{array}$ & $\begin{array}{l}13,615.15 \\
13,616.52 \\
13,640.06 \\
13,701.66 \\
13,776.71\end{array}$ & $\begin{array}{l}4 p^{4} \mathrm{D}_{11 / 2}^{\circ}-6 s^{4} \mathrm{P}_{01 / 2} \\
4 p^{2} \mathrm{P}_{01 / 2}^{0}-4 d^{2} \mathrm{P}_{11 / 2} \\
4 p^{4} \mathrm{D}_{31 / 2}^{1}-4 d^{2} \mathrm{D}_{21 / 2} \\
4 s^{4} \mathrm{P}_{21 / 2}-4 p^{4} \mathrm{~S}_{11 / 2}^{\circ}\end{array}$ \\
\hline $\begin{array}{l}7,252.48 \\
7,244.76 \\
7,194.94 \\
7,185.68 \\
7,146.38\end{array}$ & $\begin{array}{l}3 \\
3 \\
5 \\
2 \\
5\end{array}$ & $\begin{array}{l}13,784.60 \\
13,799.29 \\
13,894.83 \\
13,912.74 \\
13,989.25\end{array}$ & $\begin{array}{l}4 p^{4} \mathrm{~S}_{11 / 2}-4 d^{2} \mathrm{P}_{11 / 2} \\
4 p^{4} \mathrm{P}_{01 / 2}^{\circ}-4 d^{4} \mathrm{~F}_{11 / 2} \\
4 p^{4} \mathrm{D}_{21 / 2}^{\circ}-4 d^{4} \mathrm{P}_{11 / 2}^{\circ}\end{array}$ \\
\hline $\begin{array}{l}7,127.35 \\
7,125.57 \\
7,094.20 \\
7,086.80 \\
7,082.35\end{array}$ & $\begin{array}{r}3 \\
2 \\
8 \\
25 \\
3\end{array}$ & $\begin{array}{l}14,026.60 \\
14,030.10 \\
14,092.14 \\
14,106.86 \\
14,115.72\end{array}$ & $\begin{array}{l}4 p^{4} \mathrm{P}_{21 / 2}^{\circ}-4 d^{4} \mathrm{~F}_{21 / 2} \\
{ }^{4} p^{2} \mathrm{P}_{11 / 2}^{\circ}-4 d^{2} \mathrm{P}_{11 / 2} \\
4 p^{4} \mathrm{P}_{11 / 2}^{\circ}-6 s^{4} \mathrm{P}_{21 / 2} \\
4 p^{4} \mathrm{P}_{01 / 2}^{\circ}-6 s^{4} \mathrm{P}_{11 / 2}^{\circ}\end{array}$ \\
\hline $\begin{array}{l}7,075.64 \\
7,060.18 \\
7,058.25 \\
7,036.30 \\
7,021.99\end{array}$ & $\begin{array}{l}3 \\
2 \\
1 \\
5 \\
1\end{array}$ & $\begin{array}{l}14,129.10 \\
14,160.05 \\
14,163.90 \\
14,208.10 \\
14,237.06\end{array}$ & $\begin{array}{l}4 p^{4} \mathrm{P}_{11 / 2}-4 d^{4} \mathrm{~F}_{11 / 2} \\
4 p^{4} \mathrm{D}_{11 / 2}^{\circ}-4 d^{4} \mathrm{P}_{01 / 2} \\
4 p^{2} \mathrm{~S}_{01 / 2}^{\circ}-5 d^{4} \mathrm{D}_{01 / 2} \\
4 p^{4} \mathrm{P}_{11 / 2}^{\circ}-4 d^{4} \mathrm{P}_{21 / 2}^{\circ}\end{array}$ \\
\hline $\begin{array}{l}7,019.30 \\
7,011.24 \\
7,008.00 \\
7,006.30 \\
6,995.88\end{array}$ & $\begin{array}{r}4 \\
3 \\
10 \\
4 \\
12\end{array}$ & $\begin{array}{l}14,242.51 \\
14,258.89 \\
14,265.48 \\
14,268.94 \\
14,290.19\end{array}$ & $\begin{array}{l}4 p^{4} \mathrm{P}_{21 / 2}^{\circ}-4 d^{2} \mathrm{~F}_{21 / 2} \\
4 p^{2} \mathrm{P}_{11 / 2}^{\circ}-4 d^{2} \mathrm{P}_{01 / 2} \\
4 p^{2} \mathrm{~S}_{01 / 2}^{\circ}-4 d^{2} \mathrm{P}_{11 / 2}\end{array}$ \\
\hline $\begin{array}{l}6,981.85 \\
6,979.60 \\
6,977.00 \\
6,966.80 \\
6,962.50\end{array}$ & $\begin{array}{r}25 \\
3 \\
5 \\
8 \\
6\end{array}$ & $\begin{array}{l}14,318.91 \\
14,323.52 \\
14,328.86 \\
14,349.84 \\
14,358.70\end{array}$ & $\begin{array}{l}4 p^{4} \mathrm{P}_{21 / 2}^{\circ}-6 s^{4} \mathrm{P}_{21 / 2} \\
4 p^{2} \mathrm{P}_{11 / 2}^{\circ}-5 d^{4} \mathrm{~F}_{21 / 2} \\
4 p^{4} \mathrm{P}_{11 / 2}^{\circ}-6 s^{4} \mathrm{P}_{11 / 2}\end{array}$ \\
\hline
\end{tabular}


TABLE 1.-Wave lengths in the arc spectrum of chlorine-Continued

\begin{tabular}{|c|c|c|c|}
\hline$\lambda_{\text {sir. I. A. }}$ & Intensity & $\nu_{\mathrm{VAO}} \mathrm{cm}^{-1}$ & Term combination \\
\hline $\begin{array}{l}6,932.90 \\
6,925.35 \\
6,924.40 \\
6,910.32 \\
6,872.85\end{array}$ & $\begin{array}{r}25 \\
6 \\
5 \\
6 \\
6\end{array}$ & $\begin{array}{l}14,420.00 \\
14,435.73 \\
14,437.71 \\
14,467.02 \\
14,546.00\end{array}$ & $\begin{array}{l}4 p^{4} \mathrm{P}_{21 / 5}^{\circ}-4 d^{4} \mathrm{P}_{21 / 5} \\
4 p^{2} \mathrm{~S}_{01 / 5}^{\circ}-4 d^{2} \mathrm{P}_{01 / 5} \\
4 p^{2} \mathrm{D}_{1 / 5}^{\circ}-4 d^{2} \mathrm{P}_{1 / 5}\end{array}$ \\
\hline $\begin{array}{l}6,865.36 \\
6,854.45 \\
6,841.74 \\
6,840.23 \\
6,837.60\end{array}$ & $\begin{array}{r}8 \\
10 \\
6 \\
15 \\
5\end{array}$ & $\begin{array}{l}14,561.87 \\
14,585.05 \\
14,612.14 \\
14,615.37 \\
14,620.99\end{array}$ & $\begin{array}{l}4 p^{4} \mathrm{P}_{21 / 5}^{\circ}-6 s^{4} \mathrm{P}_{11 / 5} \\
4 p^{4} \mathrm{~S}_{1 / 5}^{\circ}-5 d^{2} \mathrm{D}_{11 / 5} \\
4 p^{4} \mathrm{P}_{21 / 5}^{\circ}-4 d^{2} \mathrm{D}_{21 / 5} \\
4 p^{2} \mathrm{D}_{23 / 5}^{2}-5 d^{4} \mathrm{D}_{21 / 5}\end{array}$ \\
\hline $\begin{array}{l}6,816.50 \\
6,811.50 \\
6,810.04 \\
6,791.92 \\
6,790.20\end{array}$ & $\begin{array}{r}2 \\
3 \\
15 \\
3 \\
2\end{array}$ & $\begin{array}{l}14,666.24 \\
14,677.01 \\
14,680.23 \\
14,719.32 \\
14,723.05\end{array}$ & $\begin{array}{l}4 p^{4} \mathrm{D}_{01 / 5}^{\circ}-5 d^{4} \mathrm{D}_{11 / 5} \\
4 p^{4} \mathrm{P}_{1 / 2}^{\circ}-4 d^{2} \mathrm{D}_{11 / 5} \\
4 p^{4} \mathrm{P}_{01 / 5}^{\circ}-4 d^{4} \mathrm{P}_{11 / 5} \\
4 p^{4} \mathrm{D}_{01 / 2}^{\circ}-5 d^{4} \mathrm{D}_{01 / 2} \\
4 p^{2} \mathrm{D}_{1 / 1 / 5}^{\circ}-4 d^{2} \mathrm{P}_{01 / 2}\end{array}$ \\
\hline $\begin{array}{l}6,784.45 \\
6,765.20 \\
6,762.30 \\
6,760.05 \\
6,757.75\end{array}$ & $\begin{array}{l}1 \\
3 \\
2 \\
2 \\
5\end{array}$ & $\begin{array}{l}14,735.53 \\
14,777.46 \\
14,783.80 \\
14,788.82 \\
14,793.75\end{array}$ & $\begin{array}{l}4 p^{4} \mathrm{P}_{01 / 5}^{\circ}-6 s^{4} \mathrm{P}_{01 / 5} \\
4 p^{2} \mathrm{D}_{1 / 5}^{1}-5 d^{4} \mathrm{~F}_{21 / 5} \\
4 p^{4} \mathrm{D}_{1 / 5}^{\circ}-5 d^{4} \mathrm{D}_{21 / 5}\end{array}$ \\
\hline $\begin{array}{l}6,751.54 \\
6,730.24 \\
6,723.40 \\
6,714.65 \\
6,709.90\end{array}$ & $\begin{array}{l}4 \\
5 \\
4 \\
4 d ? \\
15\end{array}$ & $\begin{array}{l}14,807.27 \\
14,854.22 \\
14,869.33 \\
14,888.71 \\
14,899.25\end{array}$ & $\begin{array}{l}4 p^{2} \mathrm{P}_{11 / 5}^{\circ}-5 d^{2} \mathrm{D}_{21 / 2} \\
4 p^{4} \mathrm{~S}_{11 / 5}-5 d^{2} \mathrm{~F}_{21 / 5} \\
4 p^{4} \mathrm{D}_{1 / 5 /}^{\circ}-5 d^{4} \mathrm{D}_{11 / 5} \\
4 p^{4} \mathrm{P}_{21 / 5}^{\circ}-4 d^{2} \mathrm{D}_{11 / 2}\end{array}$ \\
\hline $\begin{array}{l}6,703 \cdot 20 \\
6,699.40 \\
6,697.45 \\
6,678.39 \\
6,653.80\end{array}$ & $\begin{array}{r}6 \\
3 \\
2 \\
10 \\
1\end{array}$ & $\begin{array}{l}14,914.14 \\
14,922.60 \\
14,926.94 \\
14,969.54 \\
15,024.86\end{array}$ & $\begin{array}{l}4 p^{4} \mathrm{P}_{1 / 5}-4 d^{4} \mathrm{P}_{11 / 5} \\
4 p^{4} \mathrm{D}_{1 / 5}-5 d^{4} \mathrm{D}_{01 / 2} \\
4 p^{2} \mathrm{~S}_{01 / 5}^{0}-5 d^{4} \mathrm{P}_{01 / 5} \\
4 p^{4} \mathrm{P}_{11 / 5}^{\circ}-6 s^{4} \mathrm{P}_{01 / 5}^{\circ}\end{array}$ \\
\hline $\begin{array}{l}6,643.10 \\
6,637.15 \\
6,634.60 \\
6,632.75 \\
6,611.32\end{array}$ & $\begin{array}{l}1 \\
2 \\
0 \\
0 \\
1\end{array}$ & $\begin{array}{l}15,049.06 \\
15,062.55 \\
15,068.34 \\
15,072.55 \\
15,121.40\end{array}$ & $\begin{array}{l}4 p^{4} \mathrm{D}_{1 \zeta \zeta}-4 d^{2} \mathrm{P}_{11 / 5} \\
4 p^{4} \mathrm{D}_{2 \zeta \zeta}^{\circ}-5 d^{4} \mathrm{D}_{31 / 3}\end{array}$ \\
\hline $\begin{array}{l}6,609.26 \\
6,604.57 \\
6,600.10 \\
6,567.35 \\
6,565.90\end{array}$ & $\begin{array}{l}7 \\
7 \\
3 \\
3 \\
1\end{array}$ & $\begin{array}{l}15,126.12 \\
15,136.86 \\
15,147.11 \\
15,222.64 \\
15,226.01\end{array}$ & $\begin{array}{l}4 p^{4} \mathrm{P}_{21 / 2}^{\circ}-4 d^{4} \mathrm{P}_{11 / 2} \\
4 p^{4} \mathrm{D}_{21 / 2}^{\circ}-5 d^{4} \mathrm{D}_{21 / 2} \\
4 p^{2} \mathrm{P}_{1 / 2}^{\circ}-5 d^{2} \mathrm{~F}_{21 / 2} \\
4 p^{4} \mathrm{D}_{21 / 2}^{\circ}-5 d^{4} \mathrm{D}_{11 / 2} \\
4 p^{4} \mathrm{D}_{11 / 2}^{\circ}-4 d^{2} \mathrm{P}_{01 / 2}\end{array}$ \\
\hline $6,551.28$ & 3 & $15,259.98$ & \\
\hline $6,550.80$ & 3 & $15,261.10$ & $\left\{\begin{array}{l}4 p^{2} D_{1 / 3}-5 d^{2} D_{23 / 6} \\
4 p^{4} \mathrm{D}_{03 / 5}-5 d^{4} \mathrm{~F}_{11 / 5}\end{array}\right.$ \\
\hline $6,542.40$ & 8 & $15,280.70$ & $\left\{\begin{array}{l}4 p^{4} \mathrm{P}_{01 / 5}^{\circ}-4 d^{4} \mathrm{P}_{01 / 2} \\
4 p^{4} \mathrm{D}_{1 / 5}^{\circ}-5 d^{4} \mathrm{~F}_{21 / 2}\end{array}\right.$ \\
\hline $\begin{array}{l}6,536.10 \\
6,531.39 \\
6,528.12 \\
6,526.46 \\
6,509.00\end{array}$ & $\begin{array}{r}1 \\
20 \\
0 \\
1 \\
2\end{array}$ & $\begin{array}{l}15,295.43 \\
15,306.45 \\
15,314.12 \\
15,318.02 \\
15,359.11\end{array}$ & $\begin{array}{l}4 p^{4} \mathrm{D}_{31 / 5}^{\circ}-5 d^{4} \mathrm{D}_{31 / 5} \\
4 p^{2} \mathrm{D}_{1 / \zeta}-5 d^{2} \mathrm{D}_{1 / 2 /}\end{array}$ \\
\hline
\end{tabular}


TABLE 1.-Wave lengths in the arc spectrum of chlorine-Continued

\begin{tabular}{|c|c|c|c|}
\hline$\lambda_{\mathrm{sir}}$, I. A. & Intensity & $\nu_{\mathrm{YaO}} \mathrm{cm}^{-1}$ & Term combination \\
\hline $\begin{array}{l}6,502.21 \\
6,492.76 \\
6,490.55 \\
6,485.38 \\
6,475.90\end{array}$ & $\begin{array}{l}5 \\
0 \\
1 \\
1 \\
1\end{array}$ & $\begin{array}{l}15,375.15 \\
15,397.52 \\
15,402.77 \\
15,415.05 \\
15,437.61\end{array}$ & $\begin{array}{l}4 p^{4} \mathrm{D}_{31 / 2}^{\circ}-5 d^{4} \mathrm{D}_{21 / 2} \\
4 p^{4} \mathrm{D}_{21 / 2}^{\circ}-4 d^{2} \mathrm{P}_{11 / 2}\end{array}$ \\
\hline $\begin{array}{l}6,471.45 \\
6,464.60 \\
6,457.14 \\
6,455.24 \\
6,452.75\end{array}$ & $\begin{array}{l}3 \\
1 \\
1 \\
0 \\
1\end{array}$ & $\begin{array}{l}15,448.23 \\
15,464.60 \\
15,482.46 \\
15,487.02 \\
15,492.99\end{array}$ & $\begin{array}{l}4 p^{4} \mathrm{D}_{11 / 2}^{\circ}-5 d^{4} \mathrm{~F}_{11 / 2} \\
4 p^{4} \mathrm{D}_{01 / 2}^{\circ}-5 d^{4} \mathrm{P}_{01 / 2}^{\circ}\end{array}$ \\
\hline $\begin{array}{l}6,450.30 \\
6,443.76 \\
6,440.20 \\
6,434.79 \\
6,425.61\end{array}$ & $\begin{array}{r}12 \\
2 \\
1 \\
15 \\
8\end{array}$ & $\begin{array}{l}15,498.88 \\
15,514.61 \\
15,523.19 \\
15,536.24 \\
15,558.43\end{array}$ & $\begin{array}{l}4 p^{2} \mathrm{D}_{21 / 2}^{\circ}-5 d^{2} \mathrm{~F}_{31 / 2} \\
4 p^{4} \mathrm{P}_{11 / 2}^{\circ}-4 d^{4} \mathrm{P}_{01 / 2} \\
4 p^{4} \mathrm{D}_{21 / 2}^{\circ}-5 d^{4} \mathrm{~F}_{31 / 2}\end{array}$ \\
\hline $6,409.64$ & 0 & $15,597.20$ & \\
\hline $6,408.05$ & 7 & $15,601.07$ & $\left\{\begin{array}{l}4 p^{2} \mathrm{D}_{11 / 5}-5 d^{2} \mathrm{~F}_{21 / 2} \\
4 p^{2} \mathrm{D}_{315}-5 d^{2} \mathrm{D}^{214}\end{array}\right.$ \\
\hline $\begin{array}{l}6,398.63 \\
6,395.66\end{array}$ & $\begin{array}{r}20 \\
0\end{array}$ & $\begin{array}{l}15,624.04 \\
15,631.29\end{array}$ & $4 p^{4} \mathrm{D}_{31 / 2}^{\circ}-5 d^{4} \mathrm{~F}_{41 / 2}^{2 / 2}$ \\
\hline $\begin{array}{l}6,394.75 \\
6,376.28 \\
6,373.37 \\
6,371.40 \\
6,367.98\end{array}$ & $\begin{array}{l}4 \\
3 \\
1 \\
2 \\
3\end{array}$ & $\begin{array}{l}15,633.52 \\
15,678.80 \\
15,685.96 \\
15,690.81 \\
15,699.23\end{array}$ & $\begin{array}{l}4 p^{4} \mathrm{D}_{21 / 2}^{\circ}-5 d^{4} \mathrm{~F}_{21 / 2} \\
4 p^{4} \mathrm{D}_{11 / 2}^{\circ}-5 d^{4} \mathrm{P}_{01 / 2} \\
4 p^{2} \mathrm{D}_{21 / 2}^{\circ}-5 d^{2} \mathrm{D}_{11 / 2}\end{array}$ \\
\hline $\begin{array}{l}\text { 6, 366. } 83 \\
6,366.41 \\
6,361.19 \\
6,347.00 \\
6,342.72\end{array}$ & $\begin{array}{l}2 \\
2 \\
2 \\
4 \\
2\end{array}$ & $\begin{array}{l}15,702.07 \\
15,703.11 \\
15,715.99 \\
15,751.13 \\
15,761.76\end{array}$ & \\
\hline $\begin{array}{l}6,341.66 \\
6,337.59 \\
6,334.96 \\
6,329.65 \\
6,326.74\end{array}$ & $\begin{array}{r}10 \\
0 \\
4 \\
0 \\
3\end{array}$ & $\begin{array}{l}15,764.39 \\
15,774.51 \\
15,781.06 \\
15,794.30 \\
15,801.57\end{array}$ & $\begin{array}{l}4 p^{4} \mathrm{D}_{11 / 3}^{1}-5 d^{2} \mathrm{D}_{21 / 5} \\
4 p^{4} \mathrm{D}_{3 ! 5}^{2}-5 d^{4} \mathrm{~F}_{31 / 2}\end{array}$ \\
\hline $\begin{array}{l}6,321.59 \\
6,304.94 \\
6,280.20 \\
6,276.50 \\
6,274.51\end{array}$ & $\begin{array}{l}6 \\
0 \\
5 \\
1 \\
0\end{array}$ & $\begin{array}{l}15,814.44 \\
15,856.20 \\
15,918.67 \\
15,928.05 \\
15,933.10\end{array}$ & $4 p^{4} \mathrm{D}_{21 / 2}^{\circ}-5 d^{4} \mathrm{P}_{21 / 2}$ \\
\hline $\begin{array}{l}\text { 6, 252. } 26 \\
6,242.54 \\
6,231.48 \\
6,228.68 \\
6,226.39\end{array}$ & $\begin{array}{l}8 \\
4 \\
8 \\
1 d ? \\
3\end{array}$ & $\begin{array}{l}15,989.80 \\
16,014.70 \\
16,043.12 \\
16,050.34 \\
16,056.24\end{array}$ & $\begin{array}{l}4 p^{4} \mathrm{P}_{01 / 3}^{0}-5 d^{4} \mathrm{D}_{11 / 2} \\
4 p^{4} \mathrm{D}_{21 / 5}^{2}-5 d^{2} \mathrm{~F}_{31 / 6} \\
4 p^{4} \mathrm{P}_{01 / 2}^{1}-5 d^{4} \mathrm{D}_{01 / 2}\end{array}$ \\
\hline $\begin{array}{l}6,211.55 \\
6,194.72 \\
6,165.38 \\
6,162.05 \\
6,159.80\end{array}$ & $\begin{array}{r}6 \\
15 \\
1 \\
12 \\
0\end{array}$ & $\begin{array}{l}16,094.60 \\
16,138.32 \\
16,215.12 \\
16,223.89 \\
16,229.81\end{array}$ & $\begin{array}{l}4 p^{4} \mathrm{D}_{31 / 2}^{\circ}-5 d^{4} \mathrm{P}_{21 / 2} \\
4 p^{4} \mathrm{P}_{11 / 2}-5 d^{4} \mathrm{D}_{21 / 2} \\
4 p^{4} \mathrm{D}_{21 / 2}^{0}-5 d^{2} \mathrm{D}_{11 / 2} \\
4 p^{4} \mathrm{P}_{11 / 2}^{\circ}-5 d^{4} \mathrm{D}_{11 / 2}\end{array}$ \\
\hline
\end{tabular}


TABLE 1.-Wave lengths in the arc spectrum of chlorine-Continued

\begin{tabular}{|c|c|c|c|}
\hline$\lambda_{\mathrm{a} \text { ir. I. A. }}$ & Intensity & $\nu_{\text {raO }} \mathrm{cm}^{-1}$ & Term combination \\
\hline $\begin{array}{l}6,157.85 \\
6,151.34 \\
6,141.79 \\
6,140.21 \\
6,127.00\end{array}$ & $\begin{array}{r}2 \\
2 \\
4 \\
25 \\
0\end{array}$ & $\begin{array}{l}16,234.95 \\
16,252.13 \\
16,277.40 \\
16,281.59 \\
16,316.70\end{array}$ & $\begin{array}{l}4 p^{4} \mathrm{D}_{31 / 2}^{\circ}-5 d^{2} \mathrm{~F}_{31 / 2} \\
4 p^{4} \mathrm{P}_{11 / 3}-5 d^{4} \mathrm{D}_{01 / 2} \\
4 p^{4} \mathrm{P}_{21 / 3}^{\circ}-5 d^{4} \mathrm{D}_{31 / 2}\end{array}$ \\
\hline $\begin{array}{l}6,114.37 \\
6,110.38 \\
6,082.53 \\
6,076.45 \\
6,073.39\end{array}$ & $\begin{array}{r}15 \\
1 \\
4 \\
1 \\
1\end{array}$ & $\begin{array}{l}16,350.77 \\
16,361.07 \\
16,435.99 \\
16,452.43 \\
16,460.72\end{array}$ & $\begin{array}{l}4 p^{4} \mathrm{P}_{21 / 2}^{\circ}-5 d^{4} \mathrm{D}_{21 / 2} \\
4 p^{4} \mathrm{P}_{21 / 2}^{\circ}-5 d^{4} \mathrm{D}_{11 / 2}\end{array}$ \\
\hline $\begin{array}{l}6,058 \cdot 33 \\
6,042 \cdot 34 \\
6,040.43 \\
6,037.14 \\
6,019.71\end{array}$ & $\begin{array}{l}0 \\
1 \\
2 \\
1 \\
6\end{array}$ & $\begin{array}{l}16,501.64 \\
16,545.31 \\
16,550.51 \\
16,559.56 \\
16,607.51\end{array}$ & \\
\hline $\begin{array}{l}6,003.03 \\
5,991.42 \\
5,972.75 \\
5,969.86 \\
5,965.71\end{array}$ & $\begin{array}{l}0 \\
4 \\
2 \\
0 \\
2\end{array}$ & $\begin{array}{l}16,653.73 \\
16,685.92 \\
16,738.08 \\
16,746.18 \\
16,757.83\end{array}$ & 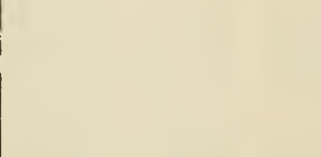 \\
\hline $\begin{array}{l}5,948.40 \\
5,936.20 \\
5,934.00\end{array}$ & $\begin{array}{l}4 \\
0 \\
1\end{array}$ & $\begin{array}{l}16,806.60 \\
16,841.14 \\
16,847.38\end{array}$ & $\begin{array}{l}4 p^{4} \mathrm{P}_{01 / 2}^{0}-5 d^{4} \mathrm{P}_{01 / 2} \\
4 p^{4} \mathrm{P}_{21 / 3}^{\circ}-5 d^{4} \mathrm{~F}_{21 / 2}\end{array}$ \\
\hline $5,930.35$ & 6 & $16,857.75$ & $\left\{\begin{array}{l}4 p^{4} \mathrm{D}_{21 / 2}-6 d^{4} \mathrm{D}_{31 / 2} \\
4 p^{4} \mathrm{P}_{11 / 2}^{0}-5 d^{4} \mathrm{P}_{21 / 2}\end{array}\right.$ \\
\hline $\begin{array}{l}5,916.31 \\
5,910.75 \\
5,908.22 \\
5,889.14 \\
5,880.76\end{array}$ & $\begin{array}{l}0 \\
0 \\
2 \\
1 \\
1\end{array}$ & $\begin{array}{l}16,897.76 \\
16,913.65 \\
16,920.89 \\
16,975.71 \\
16,999.90\end{array}$ & $4 p^{4} \mathrm{D}_{21 / 2}-6 d^{4} \mathrm{D}_{21 / 2}$ \\
\hline $\begin{array}{l}5,877.53 \\
5,866.75 \\
5,864.44 \\
5,861.27 \\
5,856.70\end{array}$ & $\begin{array}{l}2 \\
4 \\
0 \\
1 \\
8\end{array}$ & $\begin{array}{l}17,009.25 \\
17,040.50 \\
17,047.21 \\
17,056.43 \\
17,069.74\end{array}$ & $\begin{array}{l}4 p^{4} \mathrm{P}_{1 / 2 / 2}-5 d^{4} \mathrm{P}_{01 / 2} \\
4 p^{4} \mathrm{P}_{21 / 2}^{\circ}-5 d^{4} \mathrm{P}_{21 / 2}\end{array}$ \\
\hline $\begin{array}{l}5,847.68 \\
5,846.70 \\
5,844.15 \\
5,842.39 \\
5,839.85\end{array}$ & $\begin{array}{l}7 \\
8 \\
6 \\
1 \\
4\end{array}$ & $\begin{array}{l}17,096.07 \\
17,098.94 \\
17,106.40 \\
17,111.55 \\
17,118.99\end{array}$ & $\begin{array}{l}4 p^{4} \mathrm{D}_{31 / 2}^{\circ}-6 d^{4} \mathrm{D}_{31 / 2} \\
4 p^{4} \mathrm{P}_{1 / 2}-5 d^{4} \mathrm{P}_{11 / 2} \\
4 p^{4} \mathrm{P}_{11 / 2}^{\circ}-5 d^{2} \mathrm{D}_{21 / 2}\end{array}$ \\
\hline $\begin{array}{l}5,827.44 \\
5,826.24 \\
5,819.44 \\
5,817.58 \\
5,808.59\end{array}$ & $\begin{array}{l}2 \\
2 \\
0 \\
1 \\
1\end{array}$ & $\begin{array}{l}17,155.45 \\
17,158.98 \\
17,179.03 \\
17,184.52 \\
17,211.12\end{array}$ & $4 p^{4} \mathrm{D}_{31 / 2}^{\circ}-6 d^{4} \mathrm{D}_{21 / 2}$ \\
\hline $\begin{array}{l}5,806.76 \\
5,802.84 \\
5,799.88 \\
5,796.26 \\
5,774.72\end{array}$ & $\begin{array}{r}2 \\
5 \\
12 \\
15 \\
4\end{array}$ & $\begin{array}{l}17,216.54 \\
17,228.17 \\
17,236.97 \\
17,247.73 \\
17,312.07\end{array}$ & $\begin{array}{l}4 p^{4} \mathrm{P}_{i 1 / 5}-5 d^{2} \mathrm{D}_{11 / 2} \\
4 p^{4} \mathrm{P}_{21 / 2}^{\circ}-5 d^{2} \mathrm{~F}_{31 / 2}\end{array}$ \\
\hline
\end{tabular}


TABLE 1.-Wave lengths in the arc spectrum of chlorine-Continued

\begin{tabular}{|c|c|c|c|}
\hline$\lambda_{\mathrm{sir}}$. I. A. & Intensity & $\nu_{\mathrm{VBc}} \mathrm{cm}^{-1}$ & Term combination \\
\hline $\begin{array}{l}5,772.58 \\
5,769.36 \\
5,768.30 \\
5,765.55 \\
5,726.16\end{array}$ & $\begin{array}{l}5 \\
2 \\
2 \\
3 \\
5\end{array}$ & $\begin{array}{l}17,318.48 \\
17,328.15 \\
17,331.34 \\
17,339.60 \\
17,458.88\end{array}$ & $\begin{array}{l}4 p^{4} \mathrm{P}_{21 / 2}^{\circ}-5 d^{4} \mathrm{P}_{11 / 2} \\
4 p^{4} \mathrm{P}_{21 / 2}^{\circ}-5 d^{2} \mathrm{D}_{21 / 2} \\
4 p^{4} \mathrm{P}_{11 / 2}^{\circ}-5 d^{2} \mathrm{~F}_{21 / 2}\end{array}$ \\
\hline $\begin{array}{l}5,711.68 \\
5,702.02 \\
5,686.28 \\
5,673.01 \\
5,660.96\end{array}$ & $\begin{array}{l}1 \\
1 \\
1 \\
1 \\
2\end{array}$ & $\begin{array}{l}17,503.14 \\
17,532.79 \\
17,581.32 \\
17,622.45 \\
17,659.96\end{array}$ & \\
\hline $\begin{array}{l}5,654.20 \\
5,620.72 \\
5,582.28 \\
5,580.45 \\
5,578.13\end{array}$ & $\begin{array}{l}1 \\
1 \\
1 \\
3 \\
3\end{array}$ & $\begin{array}{l}17,681.07 \\
17,786.39 \\
17,908.87 \\
17,914.74 \\
17,922.16\end{array}$ & $4 p^{4} \mathrm{P}_{1 / 1 / 2}^{\circ}-6 d^{4} \mathrm{D}_{21 / 2}$ \\
\hline $\begin{array}{l}5,549.21 \\
5,541.00 \\
5,532.13 \\
5,512.95 \\
5,493.14\end{array}$ & $\begin{array}{l}1 \\
1 \\
8 \\
4 \\
3\end{array}$ & $\begin{array}{l}18,015.59 \\
18,042.28 \\
18,071.21 \\
18,134.08 \\
18,199.48\end{array}$ & $\begin{array}{l}4 p^{4} \mathrm{P}_{21 / 2}^{\circ}-6 d^{4} \mathrm{D}_{31 / 2} \\
4 p^{4} \mathrm{P}_{21 / 2}^{\circ}-6 d^{4} \mathrm{D}_{21 / 2}\end{array}$ \\
\hline $\begin{array}{l}5,484.60 \\
5,464.31 \\
5,401.65 \\
5,376.32 \\
5,338.00\end{array}$ & $\begin{array}{l}1 \\
1 \\
1 \\
1 \\
2\end{array}$ & $\begin{array}{l}18,227.82 \\
18,295.50 \\
18,507.73 \\
18,594.93 \\
18,728.41\end{array}$ & \\
\hline $\begin{array}{l}5,315.10 \\
5,307.55 \\
5,231.41 \\
5,140.35 \\
5,099.80\end{array}$ & $\begin{array}{l}1 \\
2 \\
2 \\
5 \\
8\end{array}$ & $\begin{array}{l}18,809.10 \\
18,835.86 \\
19,110.00 \\
19,448.52 \\
19,603.16\end{array}$ & $\begin{array}{l}4 s^{2} \mathrm{P}_{01 / 2}-4 p^{2} \mathrm{P}_{11 / 2}^{\circ},\left({ }^{3} \mathrm{P}^{1} \mathrm{D}\right) \\
4 s^{2} \mathrm{P}_{01 / 2}-4 p^{2} \mathrm{P}_{01 / 2}^{\circ},\left({ }^{3} \mathrm{P}^{1} \mathrm{D}\right)\end{array}$ \\
\hline $\begin{array}{l}5,074.25 \\
4,976.62 \\
4,952.76 \\
4,852.70 \\
4,818.64\end{array}$ & $\begin{array}{r}1 \\
10 \\
1 \\
8 \\
2\end{array}$ & $\begin{array}{l}19,701.87 \\
20,088.37 \\
20,185.14 \\
20,601.35 \\
20,746.96\end{array}$ & $\begin{array}{l}4 s^{2} \mathrm{P}_{11 / 2}-4 p^{2} \mathrm{P}_{11 / 2}^{\circ},\left({ }^{3} \mathrm{P}^{1} \mathrm{D}\right) \\
4 s^{2} \mathrm{P}_{11 / 2}-5 p^{4} \mathrm{D}_{21 / 2}^{\circ} \\
4 s^{2} \mathrm{P}_{01 / 2}-5 p^{4} \mathrm{~S}_{11 / 2}^{\circ}\end{array}$ \\
\hline $\begin{array}{l}4,818.42 \\
4,796.76 \\
4,740.71 \\
4,721.24 \\
4,691.53\end{array}$ & $\begin{array}{r}3 \\
2 \\
10 \\
8 \\
12\end{array}$ & $\begin{array}{l}20,747.91 \\
20,841.60 \\
21,088.01 \\
21,174.97 \\
21,309.07\end{array}$ & $\begin{array}{l}4 s^{2} \mathrm{P}_{11 / 2}-5 p^{4} \mathrm{P}_{01 / 2}^{\circ} \\
4 s^{2} \mathrm{P}_{01 / 2}-5 p^{2} \mathrm{D}_{11 / 2}^{\circ} \\
4 s^{2} \mathrm{P}_{11 / 2}-5 p^{4} \mathrm{D}_{11 / 2}^{\circ} \\
4 s^{2} \mathrm{P}_{11 / 2}-5 p^{2} \mathrm{D}_{21 / 2}^{\circ} \\
4 s^{2} \mathrm{P}_{11 / 2}^{\circ}-5 p^{4} \mathrm{D}_{01 / 2}^{\circ}\end{array}$ \\
\hline $\begin{array}{l}4,677.76 \\
4,674.40 \\
4,661 \cdot 22 \\
4,654.05 \\
4,633.91\end{array}$ & $\begin{array}{r}7 \\
2 \\
18 \\
10 \\
0\end{array}$ & $\begin{array}{l}21,371.79 \\
21,387.15 \\
21,447.63 \\
21,480.67 \\
21,574.03\end{array}$ & $\begin{array}{l}4 s^{2} \mathrm{P}_{11 / 2}-5 p^{2} \mathrm{~S}_{01 / 2}^{\circ} \\
4 s^{2} \mathrm{P}_{11 / 2}-5 p^{4} \mathrm{~S}_{11 / 2}^{\circ} \\
4 s^{2} \mathrm{P}_{01 / 2}-5 p^{2} \mathrm{P}_{11 / 2}^{\circ} \\
4 s^{2} \mathrm{P}_{11 / 2}-5 p^{2} \mathrm{D}_{11 / 2}^{1}\end{array}$ \\
\hline $\begin{array}{l}4,631.72 \\
4,623.96 \\
4,601.00 \\
4,580.47 \\
4,578.17\end{array}$ & $\begin{array}{r}1 \\
10 \\
20 \\
3 \\
4\end{array}$ & $\begin{array}{l}21,584.23 \\
21,620.45 \\
21,728.34 \\
21,825.72 \\
21,836.69\end{array}$ & $\begin{array}{l}4 s^{2} \mathrm{P}_{01 / 2}-4 p^{2} \mathrm{D}_{11 / 2}^{i},\left({ }^{1} \mathrm{P}^{1} \mathrm{D}\right) \\
4 s^{2} \mathrm{P}_{01 / 2}-5 p^{2} \mathrm{P}_{01 / 2} \\
4 s^{4} \mathrm{P}_{11 / 2}-4 p^{2} \mathrm{P}_{11 / 2}^{1},\left({ }^{3} \mathrm{P}^{1} \mathrm{D}\right) \\
4 s^{4} \mathrm{P}_{01 / 2}-5 p^{4} \mathrm{P}_{11 / 2}^{1}\end{array}$ \\
\hline $\begin{array}{l}4,548.06 \\
4,545.38 \\
4,526.20 \\
4,491.05 \\
4,475.31\end{array}$ & $\begin{array}{r}1 \\
0 \\
30 \\
10 \\
15\end{array}$ & $\begin{array}{l}21,981.25 \\
21,994.21 \\
22,087.42 \\
22,260.29 \\
22,338.57\end{array}$ & $\begin{array}{l}4 s^{4} \mathrm{P}_{11 / 2}-5 p^{4} \mathrm{P}_{21 / 2}^{\circ} \\
4 s^{2} \mathrm{P}_{11 / 2}-5 p^{2} \mathrm{P}_{1 / 2}^{\circ} \\
4 s^{2} \mathrm{P}_{11 / 2}-4 p^{2} \mathrm{D}_{11 / 2}^{1}, \quad\left({ }^{3} \mathrm{P}^{1} \mathrm{D}\right) \\
4 s^{4} \mathrm{P}_{11 / 2}-5 p^{4} \mathrm{D}_{21 / 2}^{\circ}\end{array}$ \\
\hline
\end{tabular}


TABLE 1.-Wave lengths in the arc spectrum of chlorine-Continued

\begin{tabular}{|c|c|c|c|}
\hline$\lambda_{\text {air. }}$ I. A. & Intensity & $\nu_{\text {vao }} \mathrm{cm}^{-1}$ & Term combination \\
\hline $\begin{array}{l}4,469.37 \\
4,446.11 \\
4,445.83 \\
4,438.48 \\
4,403.03\end{array}$ & $\begin{array}{r}18 \\
4 \\
4 \\
20 \\
15\end{array}$ & $\begin{array}{l}22,368.26 \\
22,485.28 \\
22,486.70 \\
22,523.93 \\
22,705.27\end{array}$ & $\begin{array}{l}4 s^{2} \mathrm{P}_{11 / 2}-5 p^{2} \mathrm{P}_{01 / 2}^{\circ} \\
4 s^{4} \mathrm{P}_{11 / 2}-5 p^{4} \mathrm{P}_{01 / 2}^{\circ} \\
4 s^{4} \mathrm{P}_{01 / 2}-5 p^{4} \mathrm{D}_{11 / 2}^{1} \\
4 s^{4} \mathrm{P}_{21 / 2}-5 p^{4} \mathrm{P}_{213 /}^{\circ} \\
4 s^{4} \mathrm{P}_{21 / 2}-5 p^{4} \mathrm{P}_{11 / 2}^{0}\end{array}$ \\
\hline $\begin{array}{l}4,402.58 \\
4,390.38 \\
4,389.76 \\
4,387.55 \\
4,379.90\end{array}$ & $\begin{array}{r}4 \\
7 \\
25 \\
6 \\
20\end{array}$ & $\begin{array}{l}22,707.60 \\
22,770.70 \\
22,773.91 \\
22,785.38 \\
22,825.18\end{array}$ & $\begin{array}{l}4 s^{4} \mathrm{P}_{01 / 3}-5 p^{4} \mathrm{D}_{01 / 2}^{\circ} \\
4 s^{4} \mathrm{P}_{01 / 2}-5 p^{2} \mathrm{~S}_{01 / 2}^{0} \\
4 s^{4} \mathrm{P}_{21 / 2}-5 p^{4} \mathrm{D}_{31 / 2}^{\circ} \\
4 s^{4} \mathrm{P}_{01 / 2}-5 p^{4} \mathrm{~S}_{11 / 2}^{\circ} \\
4 s^{4} \mathrm{P}_{11 / 2}-5 p^{4} \mathrm{D}_{11 / 2}^{0}\end{array}$ \\
\hline $\begin{array}{l}4,371.55 \\
4,369.52 \\
4,363.30 \\
4,337.80\end{array}$ & $\begin{array}{r}5 \\
15 \\
20 \\
1\end{array}$ & $\begin{array}{l}22,868.78 \\
22,879.40 \\
22,912.02 \\
23,046.70\end{array}$ & $\begin{array}{l}4 s^{4} \mathrm{P}_{21 / 2}-5 p^{4} \mathrm{D}_{21 / 2}^{\circ} \\
4 s^{4} \mathrm{P}_{01 / 2}-5 p^{2} \mathrm{D}_{11 / 2}^{\circ} \\
4 s^{4} \mathrm{P}_{11 / 2}-5 p^{2} \mathrm{D}_{21 / 2}^{\circ} \\
4 s^{4} \mathrm{P}_{11 / 2}-5 p^{4} \mathrm{D}_{01 / 2}^{0}\end{array}$ \\
\hline $\begin{array}{l}4,323.35 \\
4,305.55 \\
4,280.43 \\
4,264.59\end{array}$ & $\begin{array}{r}20 \\
1 \\
2 \\
5\end{array}$ & $\begin{array}{l}23,123.73 \\
23,219.33 \\
23,355.59 \\
23,442.34\end{array}$ & $\begin{array}{l}4 s^{4} \mathrm{P}_{11 / 2}-5 p^{4} \mathrm{~S}_{11 / 2} \\
4 s^{4} \mathrm{P}_{01 / 2}-5 p^{2} \mathrm{D}_{11 / 2}^{\circ} \\
4 s^{4} \mathrm{P}_{21 / 2}-5 p^{4} \mathrm{D}_{11 / 2}^{1} \\
4 s^{4} \mathrm{P}_{21 / 2}-5 p^{2} \mathrm{D}_{21 / 2}^{2}\end{array}$ \\
\hline $\begin{array}{l}4,226.44 \\
4,209.68 \\
4,147.20 \\
4,139.00\end{array}$ & $\begin{array}{r}15 \\
12 \\
2 \\
1\end{array}$ & $\begin{array}{l}23,653.94 \\
23,748.11 \\
24,105.88 \\
24,153.64\end{array}$ & $\begin{array}{l}4 s^{4} \mathrm{P}_{21 / 2}-5 p^{4} \mathrm{~S}_{11 / 2} \\
4 s^{4} \mathrm{P}_{21 / 2}-5 p^{2} \mathrm{D}_{11 / 2} \\
4 s^{4} \mathrm{P}_{11 / 2}-5 p^{2} \mathrm{P}_{01 / 2}\end{array}$ \\
\hline $\begin{array}{l}4,104.78 \\
4,032.14 \\
3,992.81 \\
3,944.79\end{array}$ & $\begin{array}{l}3 \\
5 \\
2 \\
3\end{array}$ & $\begin{array}{l}24,354.99 \\
24,793.75 \\
25,037.96 \\
25,342.74\end{array}$ & $4 s^{4} \mathrm{P}_{21 / 2}-5 p^{2} \mathrm{P}_{11 / 2}^{\circ}$ \\
\hline
\end{tabular}

$d=$ double.

A detailed description of the theoretical term structure of neutral chlorine is given in RP73. All the prominent lines of the spectrum belong to three families of terms which have ${ }^{3} \mathrm{P},{ }^{1} \mathrm{D}$, and ${ }^{1} \mathrm{~S}$ of $\mathrm{Cl}$ II as limits. The terms of these families arise when one of the $p$ electrons of the configuration $3 s^{2} .3 p^{5}$ becomes an $n s, n p$, nd ... electron in the process of excitation. A much less conspicuous group of terms will arise when one of the $s$ electrons is removed from the configuration $3 s^{2} .3 p^{5}$ and approaches ${ }^{3} \mathrm{P}^{\circ}$ or ${ }^{1} \mathrm{P}^{\circ}$ from the configuration $3 s .3 p^{5}$ of $\mathrm{Cl}$ II as limits. In table 2 are given the term groups resulting from the excitation processes just outlined.

TABLE 2.-Theoretical terms of $\mathrm{Cl} \mathrm{I}$

\begin{tabular}{|c|c|c|c|c|}
\hline \multirow{2}{*}{$\begin{array}{l}\text { Electron } \\
\text { configura- } \\
\text { tions }\end{array}$} & \multicolumn{4}{|c|}{ Limiting terms of $\mathrm{Cl}$ II } \\
\hline & ${ }^{3} \mathrm{P}$ & ${ }^{1} \mathrm{D}$ & ${ }^{1 S}$ & ${ }^{3} \mathrm{P}^{\circ},{ }_{1} \mathrm{P}^{\circ}$ \\
\hline $\begin{array}{l}3 s^{2} \cdot 3 p^{5} \\
3 s^{2} \cdot 3 p^{4} \cdot n s \\
3 s^{2} \cdot 3 p^{4} \cdot n p \\
3 s^{2} \cdot 3 p^{4} \cdot n d \\
3 s \cdot 3 p^{6}\end{array}$ & 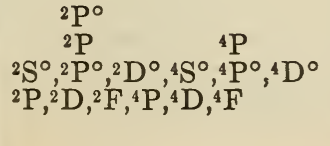 & $\begin{array}{c}{ }^{2} \mathrm{D} \\
{ }^{2} \mathrm{P}^{\circ},{ }^{2} \mathrm{D}^{\circ},{ }^{2} \mathrm{~F}^{\circ} \\
{ }^{2} \mathrm{~S},{ }^{2} \mathrm{P}^{\prime},{ }^{2} \mathrm{D},{ }^{2} \mathrm{~F},{ }^{2} \mathrm{G}\end{array}$ & $\begin{array}{l}{ }^{2} \mathrm{~S} \\
{ }^{2} \mathrm{P}^{\circ} \\
{ }^{2} \mathrm{D}\end{array}$ & ${ }^{2} \mathrm{~S}$ \\
\hline
\end{tabular}


The revised list of $\mathrm{Cl} \mathrm{I}$ terms is given in table 3. Comparison with the corresponding table of RP73 will show the following corrections and extensions. New values have been assigned to the ${ }^{2} \mathrm{~S}^{\circ}$ terms of the $4 p$ and $5 p$ groups, which otherwise remain unchanged; the nd groups, in which only the ${ }^{4} \mathrm{D}$ term had previously been established with certainty, are now practically completed, with the finding of the ${ }^{4} \mathrm{~F}$ and ${ }^{4} \mathrm{P}$ terms, and also the corresponding doublet terms; the terms with $\Delta \nu=1.70$, previously provided with the symbol ${ }^{2} \mathrm{X}$, are now definitely designated as $4 s^{2} \mathrm{D}$ belonging to the ${ }^{1} \mathrm{D}$ family. With the terms now established for $\mathrm{Cl}$ I a total of 269 lines, or 61 percent of those given in table 1, has been classified. This is approximately twice the number classified in RP73.

It should be noticed that the total quantum numbers of the nd electrons have been increased by 1 . Although the terms coming from the $3 d$ electron have not been established, yet there is good reason to believe that they are not of the same order of stability as those of the $4 s$ electron. deBruin's ${ }^{4}$ analysis of singly ionized argon, which is isoelectronic with neutral chlorine, shows that the $3 d$ terms have nearly the same values as those of the $4 s$ electron; but in the arc spectrum of argon the $3 d$ terms lie about $20,000 \mathrm{~cm}^{-1}$ higher than the $4 s$ terms. Similarly, in $\mathrm{Cl}$ Ir the analysis of Kiess and deBruin ${ }^{5}$ shows that the $3 d$ terms are next in stability after the $4 s$ terms; but if the $\mathrm{Cl}$ I terms of the $n d$ sequences are approximately represented by Rydberg's formula then those of the $3 d$ electron should fall close to the level of the $4 p$ terms, near $18,000 \mathrm{~cm}^{-1}$, in which case the lines through which they must be established will lie in the infrared beyond the limit reached in table 1.

TABLE 3.-Observed terms of $\mathrm{Cl} \mathrm{I}$

\begin{tabular}{|c|c|c|c|c|c|c|c|}
\hline $\begin{array}{l}\text { Term } \\
\text { type }\end{array}$ & $\nu$ & $\Delta \nu$ & $\begin{array}{l}\text { Series } \\
\text { electron }\end{array}$ & $\begin{array}{l}\text { Term } \\
\text { type }\end{array}$ & $\nu$ & $\Delta \nu$ & $\begin{array}{c}\text { Series } \\
\text { electron }\end{array}$ \\
\hline${ }^{2} \mathrm{P}_{\mathrm{i} / 3 / 3}$ & 104,991 & 881 & $3 n$ & ${ }^{4} \mathrm{D}_{31 / 2}^{\circ}$ & $21,101.36$ & 22826 & \\
\hline${ }^{2} \mathrm{P}_{01 / 2}^{\circ}$ & 104,110 & 001 & op & ${ }^{4} \mathrm{D}_{21 / 2}^{\circ}$ & $20,863.10$ & 200.20 & \\
\hline${ }^{4} \mathrm{P}_{23 / 2}$ & $38,037.00$ & & & ${ }^{4} D_{1 / 2}{ }^{1}$ & $20,510.09$ & 353. 01 & \\
\hline${ }^{4} \mathrm{P}_{13 / 2}$ & $32,506.80$ & 530.20 & & ${ }^{4} \mathrm{D}_{0 \% / 2}^{\circ}$ & $20,306.73$ & 203. 36 & \\
\hline${ }^{4} \mathrm{P}_{03 / 2}$ & $32,168.36$ & 508.44 & $4 s+{ }^{3} \mathrm{P}$ & ${ }^{2} \mathrm{D}_{21 / 2}^{\circ}$ & $20,347.31$ & 24025 & 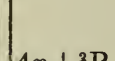 \\
\hline${ }^{2} \mathrm{P}_{13 / 2}$ & $30,769.56$ & 63980 & & ${ }^{2} \mathrm{D}_{1 / 3}^{\circ}$ & $20,006.96$ & 040.00 & $x^{ \pm p}+1$ \\
\hline${ }^{2} \mathrm{P}_{01 / 2}$ & $30,129.76$ & & & ${ }^{2} \mathrm{~S}_{01 / 3}^{\circ}$ & $19,751.02$ & & \\
\hline${ }^{2} \mathrm{D}_{21 / 2}$ & $20,875.32$ & 1.70 & $4 s+1 \mathrm{D}$ & ${ }^{2} \mathrm{P}_{11 / 2}^{\circ}$ & $19,552.96$ & $475 \quad 40$ & \\
\hline${ }^{2} \mathrm{D}_{1 / 2}$ & $20,873.62$ & & 1001 & ${ }^{2} \mathrm{P}_{03 / 3}^{\circ}$ & $19,077.56$ & 260 & \\
\hline${ }^{4} \mathrm{P}_{31 / 2}$ & $22,076.46$ & & & ${ }^{4} S_{1, \sqrt{2}}$ & $19,260.32$ & & \\
\hline${ }^{4} \mathrm{P}_{13 / 3}$ & $21,864.41$ & 233. 96 & $4 p+{ }^{3} \mathrm{P}$ & ${ }^{2} \mathrm{P}_{1 / 3}$ & $10,681.33$ & 154. 83 & $4 p+1 \mathrm{D}$ \\
\hline${ }^{4} \mathrm{P}_{01 / 2}^{\circ}$ & $21,630.45$ & & & ${ }^{2} \mathrm{P}_{03 / 5}$ & $10,526.50$ & & \\
\hline
\end{tabular}

- Proc. Acad. Sci. Amsterdam, vol. 31, p. 771, 1928; vol. 33, p. 198, 1930.

Soon to be published in Bureau of Standards Journal of Research. 
TABLE 3.-Observed ternis of $\mathrm{Cl} \mathrm{I}$-Continued

\begin{tabular}{|c|c|c|c|c|c|c|c|}
\hline $\begin{array}{l}\text { Term } \\
\text { typo }\end{array}$ & v & $\Delta \nu$ & $\begin{array}{l}\text { Series } \\
\text { electron }\end{array}$ & $\begin{array}{l}\text { Term } \\
\text { type }\end{array}$ & $\nu$ & $\Delta \nu$ & $\begin{array}{l}\text { Series } \\
\text { electron }\end{array}$ \\
\hline${ }^{2} F_{31 / 3}$ & $9,815.00$ & 3505 & & ${ }^{4} \mathrm{P}_{01 / 2}$ & $6,349.78$ & & \\
\hline${ }^{2} \mathrm{~F}_{21 / 3}$ & $9,850.95$ & & $1 n+1 n$ & ${ }^{2} \mathrm{~F}_{33 / 3}$ & $8,161.15$ & & \\
\hline${ }^{2} \mathrm{D}_{21 / 4}$ & $8,512.62$ & 332 & ע & ${ }^{2} \mathrm{~F}_{21 / 6}$ & $7,811.06$ & 300.09 & \\
\hline${ }^{2} \mathrm{D}_{\mathrm{i} / \mathrm{s}}$ & $8,509.30$ & & & ${ }^{2} \mathrm{D}_{21 / 3}$ & $7,461.15$ & 27361 & $4 d+{ }^{3} \mathrm{P}$ \\
\hline${ }^{4} \mathrm{P}_{213 /}^{\circ}$ & $10,513.07$ & 18135 & & ${ }^{2} \mathrm{D}_{11 / 3}$ & $7,187.54$ & 280.01 & \\
\hline${ }^{4} \mathrm{P}_{1 / 3}$ & $10,331.72$ & 101.00 & & ${ }^{2} \mathrm{P}_{13 / 3}$ & $5,460.90$ & & \\
\hline${ }^{4} \mathrm{P}_{035}$ & $10,021.57$ & $8+0.10$ & & ${ }^{2} \mathrm{P}_{03 / 6}$ & $5,284.00$ & 180.50 & \\
\hline${ }^{4} \mathrm{D}_{31 / 6}^{\circ}$ & $10,263.09$ & 0484 & & ${ }^{4} \mathrm{P}_{23 / 3}$ & $7,757.63$ & 24082 & \\
\hline${ }^{4} D_{213 / 2}^{\circ}$ & $10,168.25$ & 486.68 & & ${ }^{4} \mathrm{P}_{13 / 5}$ & $7,514.80$ & 61076 & $6 s+{ }^{3} \mathrm{P}$ \\
\hline${ }^{4} \mathrm{D}_{13 / 2}^{\circ}$ & $9,681.57$ & 22108 & & ${ }^{4} \mathrm{P}_{03 / 3}$ & $6,895.04$ & 017.80 & \\
\hline${ }^{4} \mathrm{D}_{01 / 3}$ & $9,460.49$ & & $5 p+{ }^{3} \mathrm{P}$ & ${ }^{4} D_{31 / 3}$ & $5,794.98$ & & \\
\hline${ }^{2} \mathrm{D}_{231 / 4}^{\circ}$ & $9,594.69$ & 30570 & & ${ }^{4} D_{21 / 2}$ & $5,726.29$ & & \\
\hline${ }^{2} D_{115}$ & $9,288.99$ & 000.8 & & ${ }^{4} D_{13 / 5}$ & $5,640.78$ & & \\
\hline${ }^{2} S_{035}$ & $9,397.72$ & & & ${ }^{4} \mathrm{D}_{01 / 2}$ & $5,587.39$ & 50. 59 & \\
\hline${ }^{4} S_{1 / 5}$ & $9,382.70$ & & & ${ }^{4} \mathrm{~F}_{41 / 3}$ & $5,477.32$ & & \\
\hline${ }^{2} \mathrm{P}_{11 / 3}$ & $8,682.16$ & & & ${ }^{4} F_{313}$ & $5,326.85$ & 100. 7 & \\
\hline${ }^{2} \mathrm{P}_{01 / 3}$ & $8,401.36$ & 200 & & $4 F_{21 / 3}$ & $5,229.48$ & & \\
\hline${ }^{4} D_{33 / 2}$ & $9,294.51$ & $850 ?$ & & ${ }^{4} F_{13 / 3}$ & $5,045.58$ & 100. 90 & $5 d+{ }^{3} \mathrm{P}$ \\
\hline${ }^{4} D_{213}$ & $9,208.59$ & 11075 & & ${ }^{4} \mathrm{P}_{23 / 5}$ & $5,006.70$ & 21870 & \\
\hline ' $D_{113}$ & $9,097.84$ & 98.02 & & ${ }^{4} \mathrm{P}_{13 / 6}$ & $4,758.00$ & 6588 & \\
\hline${ }^{4} D_{01 / 3}$ & $8,999.82$ & & & ${ }^{4} \mathrm{P}_{03 / 5}$ & $4,823.88$ & & \\
\hline${ }^{4} F_{41 / 3}$ & $8,500.60$ & & & ${ }^{2} \mathrm{~F}_{31 / 3}$ & $4,848.59$ & 112 87 & \\
\hline${ }^{4} \mathrm{~F}_{31 / 6}$ & $8,264.19$ & 214.49 & $4 d+{ }^{3} \mathrm{P}$ & ${ }^{2} \mathrm{~F}_{23 / 3}$ & $4,405.72$ & $7+2.08$ & \\
\hline${ }^{4} \mathrm{~F}_{23 / 3}$ & $8,049.70$ & 31425 & & ${ }^{2} \mathrm{D}_{23 / 3}$ & $4,745.68$ & 0766 & \\
\hline${ }^{4} F_{13 /}$ & $7,735.45$ & & & ${ }^{2} \mathrm{D}_{11 / 3}$ & $4,648.02$ & 50.00 & , \\
\hline${ }^{4} \mathrm{P}_{21 / 3}$ & $7,656.40$ & 706. 20 & & ${ }^{4} \mathrm{D}_{31 / 2}$ & $4,005.40$ & 62.87 & $6 d+{ }^{8} \mathrm{P}$ \\
\hline${ }^{4} \mathrm{P}_{13 / 2}$ & $6,950.20$ & 600.42 & & ${ }^{4} D_{21 / 2}$ & $3,942.53$ & & \\
\hline
\end{tabular}

\section{ACKNOWLEDGMENT}

In conclusion, it is a pleasure to acknowledge my indebtedness to Dr. E. Wichers, of the Bureau staff, who prepared the palladium chloride used in the experimental work.

Washington, April 20, 1933. 